\title{
Spin Correlations among the Charge Carriers in an Ordered Stripe Phase
}

\author{
A. T. Boothroyd, ${ }^{1, *}$ P. G. Freeman, ${ }^{1}$ D. Prabhakaran, ${ }^{1}$ A. Hiess, ${ }^{2}$ M. Enderle,${ }^{2}$ J. Kulda, ${ }^{2}$ and F. Altorfer ${ }^{3}$ \\ ${ }^{1}$ Department of Physics, Oxford University, Oxford, OX1 3PU, United Kingdom \\ ${ }^{2}$ Institut Laue-Langevin, BP 156, 38042 Grenoble CEDEX 9, France \\ ${ }^{3}$ Laboratory for Neutron Scattering, ETH Zürich and PSI Villigen, CH-5232 Villigen PSI, Switzerland
}

(Received 3 September 2003; published 15 December 2003)

\begin{abstract}
We have observed a diffuse component to the low-energy magnetic excitation spectrum of stripeordered $\mathrm{La}_{5 / 3} \mathrm{Sr}_{1 / 3} \mathrm{NiO}_{4}$ probed by neutron inelastic scattering. The diffuse scattering forms a square pattern with sides parallel and perpendicular to the stripe directions. The signal is dispersive, with a maximum energy of $\sim 10 \mathrm{meV}$. Probed at $2 \mathrm{meV}$, the scattering decreases in strength with increasing temperature, and is barely visible at $100 \mathrm{~K}$. We argue that the signal originates from dynamic, quasione-dimensional, antiferromagnetic correlations among the stripe electrons.
\end{abstract}

DOI: 10.1103/PhysRevLett.91.257201

PACS numbers: 75.40.Gb, 71.45.Lr, 75.30.Et, 75.30.Fv

The occurrence of stripe correlations in superconducting cuprates and other doped antiferromagnets is well established from experimental studies [1] following earlier theoretical work [2]. The stripe phase is characterized by a segregation of charge carriers into narrow channels that act as antiphase domain walls separating antiferromagnetic regions of the host spins. Several theoretical scenarios have been presented in which a spin gap and pairing instabilities appear among the stripe electrons, opening the door to superconductivity [3]. These findings suggest that stripes could play an important role in the physics of high- $T_{\mathrm{c}}$ superconductivity.

Given the characteristic spin and charge correlations associated with local stripe order, it is natural to search the spin excitation spectrum for clues to the relationship between stripes and superconductivity. Neutron inelastic scattering has revealed a number of interesting features in the spin dynamics of the cuprate superconductors, some of which, e.g., excitations at incommensurate wave vectors [4], have been interpreted as evidence for dynamic stripe correlations [5]. At the same time, the characteristic spin excitation spectrum of ideal stripes has been probed in studies of nonsuperconducting compounds with well-defined stripe order, especially $\mathrm{La}_{2-x} \mathrm{Sr}_{x} \mathrm{NiO}_{4+\delta}$ $[6,7]$. In none of these previous studies, however, has any evidence been found for spin correlations among the stripe electrons themselves.

In this Letter, we report neutron inelastic scattering measurements on $\mathrm{La}_{5 / 3} \mathrm{Sr}_{1 / 3} \mathrm{NiO}_{4}$ which reveal a pattern of inelastic diffuse scattering consistent with the existence of quasi-one-dimensional (1D) antiferromagnetic (AF) correlations parallel to the stripe direction. The correlations are found to be dynamic, with a maximum energy $\sim 10 \mathrm{meV}$. The results provide the first evidence for spin correlations among the charge carriers in a stripe phase.

The experiments were performed on single crystals of $\mathrm{La}_{5 / 3} \mathrm{Sr}_{1 / 3} \mathrm{NiO}_{4}$ grown by the floating-zone method [8]. Neutron inelastic scattering data were collected on the triple-axis spectrometers IN8, IN20, and IN22 at the Institut Laue-Langevin, and RITA-II at the Paul Scherrer Institut. Most of the data reported here were obtained with a fixed scattered neutron energy $E_{\mathrm{f}}=$ $14.7 \mathrm{meV}$ and with a graphite filter installed after the sample to suppress higher orders. The exception is the inelastic scan shown in Fig. 2(a) (below), which was measured with an incident energy $E_{\mathrm{i}}=14.7 \mathrm{meV}$ and with the filter before the sample. The incident and final neutron energies were selected by Bragg reflection from the (002) planes of graphite. When neutron polarization analysis was required Heusler (111) was used for the monochromator and analyzer.

Stripe ordering in $\mathrm{La}_{5 / 3} \mathrm{Sr}_{1 / 3} \mathrm{NiO}_{4}$ is observed below $T_{\mathrm{SO}} \simeq 200 \mathrm{~K}$, and consists of parallel lines of doped holes separating AF-ordered bands of $\mathrm{Ni}^{2+}$ spins $(S=1)$ [9]. The stripes run diagonally across the square lattice formed by the $\mathrm{Ni}$ sites on the $\mathrm{NiO}_{2}$ layers. As there is no reason to favor one diagonal over the other, a bulk sample contains an equal proportion of spatially separated domains with stripes along each diagonal.

Previous measurements have shown that the spin excitation spectrum of $\mathrm{La}_{5 / 3} \mathrm{Sr}_{1 / 3} \mathrm{NiO}_{4}$ is dominated by sharp propagating modes of the host $\mathrm{Ni}^{2+}$ spins [7]. These spin-wave-like excitations are highly 2D in nature, and extend in energy up to $\sim 80 \mathrm{meV}$. They disperse from the stripe superlattice zone centers, which have $2 \mathrm{D}$ wave vectors $\mathbf{Q}_{\mathrm{s}}=\left(h+\frac{1}{2}, k+\frac{1}{2}\right) \pm\left(\frac{1}{6}, \frac{1}{6}\right)$ for one stripe domain and $\left(h+\frac{1}{2}, k+\frac{1}{2}\right) \pm\left(-\frac{1}{6}, \frac{1}{6}\right)$ for the other, where $h$ and $k$ are integers indexed with respect to the body-centered tetragonal lattice (cell parameters $a=3.8 \AA$ and $c=12.7 \AA$ ).

In the present work, we explored the low-energy part of the spectrum over a more extended region of reciprocal space. Figure 1(a) shows the variation in scattered intensity over a rectangular portion of the $(h, k, 0)$ plane in reciprocal space, measured at an energy of $2.5 \mathrm{meV}$ and at a temperature of $2 \mathrm{~K}$. The region of reciprocal space covered by the measurements is shown in Fig. 1(b). The 

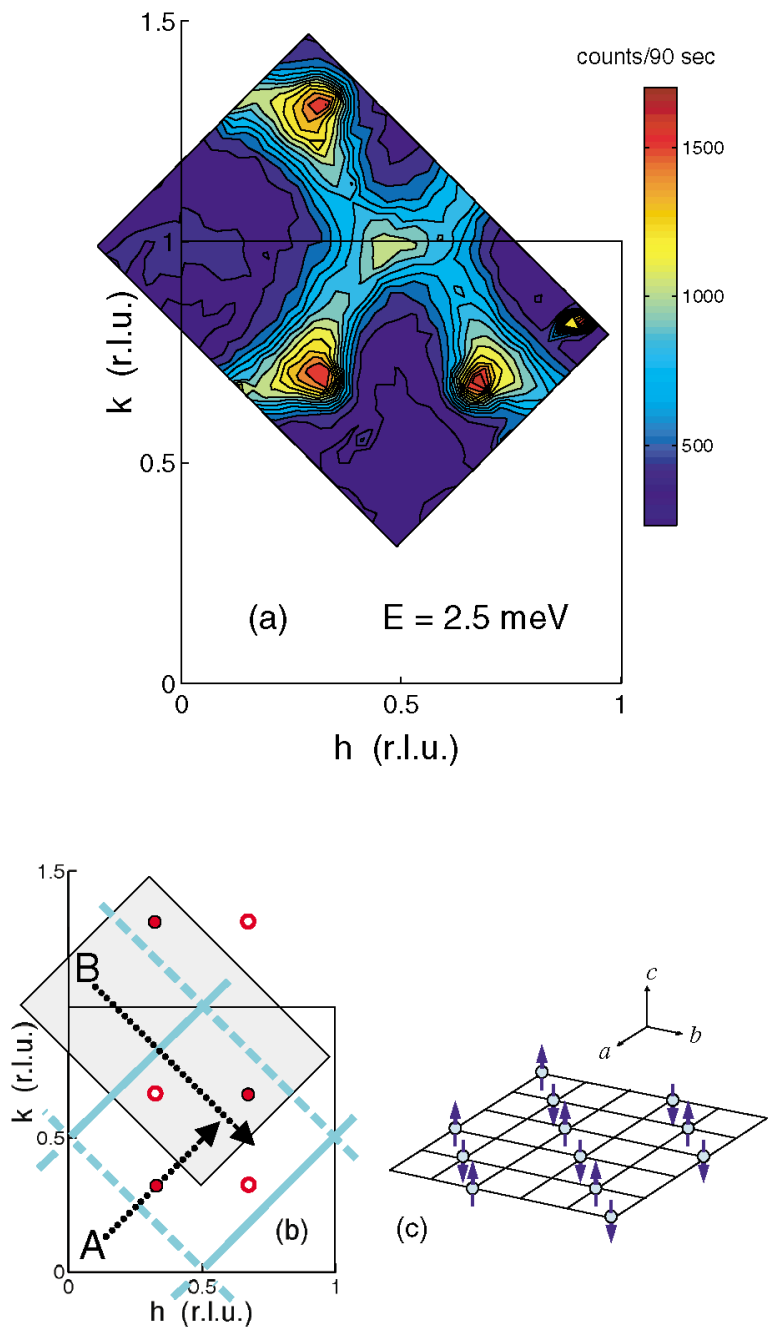

FIG. 1 (color online). Diffuse neutron inelastic scattering from $\mathrm{La}_{5 / 3} \mathrm{Sr}_{1 / 3} \mathrm{NiO}_{4}$. (a) Contour plot of the intensity in the $(h, k, 0)$ plane in reciprocal space at an energy transfer of $2.5 \mathrm{meV}$. The plot is derived from a $25 \times 35$ rectangular grid of data points. (b) Diagram of the $(h, k, 0)$ plane in reciprocal space. Circles are stripe superlattice zone centers. Diagonal lines indicate where diffuse scattering would be observed if the stripes behaved as 1D AF chains. Filled circles and full lines are for $[1,-1,0]$ stripe domains; open circles and broken lines are for $[1,1,0]$ stripe domains. The dotted lines marked A and $\mathrm{B}$ are scan directions used in the experiment. (c) $[1,1,0]$ stripe domain showing the square lattice of $\mathrm{Ni}$ sites with local 1D AF correlations on the stripes, as proposed here.

intensity map contains localized "hot spots" of intensity centered on $\mathbf{Q}_{\mathrm{S}}$ points such as $\left(\frac{1}{3}, \frac{2}{3}\right),\left(\frac{2}{3}, \frac{2}{3}\right)$, etc., marked as circles in Fig. 1(b). This scattering is from spin wave excitations of the host $\mathrm{Ni}^{2+}$ spins, as described above.

More interestingly, the data in Fig. 1(a) also show a pattern of diffuse scattering distributed around the diagonal lines drawn on Fig. 1(b). The diffuse scattering does not pass through the stripe zone centers, and is in fact separated from them. This is illustrated in Fig. 2(a), which contains two scans measured along a line equiva-
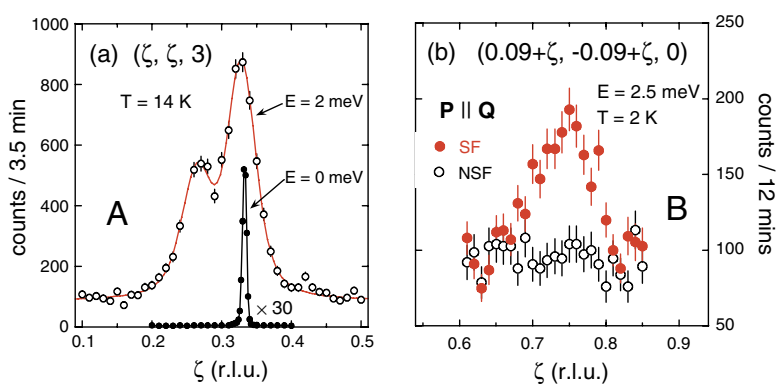

FIG. 2 (color online). Constant-energy scans along lines equivalent to those marked $\mathrm{A}$ and $\mathrm{B}$ in Fig. 1(b). The polarized-neutron data in (b) show the SF and NSF channels recorded with the neutron polarization parallel to the scattering vector.

lent to that marked A in Fig. 1(b). In the $2 \mathrm{meV}$ scan, there are two resolved peaks, one centered on $(0.27,0.27)$ from the diffuse scattering, and the other centered on $\left(\frac{1}{3}, \frac{1}{3}\right)$ from spin wave excitations of the stripe superlattice. The other scan in Fig. 2(a) records elastic scattering along the same line, and contains only the Bragg peak due to the ordered stripe superlattice. This scan shows that the diffuse scattering does not have a static component. We have also scanned the $2.5 \mathrm{meV}$ diffuse inelastic scattering parallel to the $(0,0, l)$ direction at a fixed in-plane wave vector (not shown). The intensity was found to decrease monotonically away from $l=0$ without any detectable modulation with $l$.

We next describe two measurements made with neutron polarization analysis. First, we performed scans along lines equivalent to $\mathrm{A}$ and $\mathrm{B}$ at a constant energy of $2.5 \mathrm{meV}$, recording the spin-flip (SF) and non-spin-flip (NSF) scattering with the neutron polarization $\mathbf{P}$ parallel to the scattering vector $\mathbf{Q}$. The B data are shown in Fig. 2(b). In both scans, the diffuse scattering feature was observed only in the SF channel. We conclude from this that the diffuse scattering is magnetic in origin [10].

Second, we analyzed the polarization of the $2.5 \mathrm{meV}$ diffuse scattering at two different wave vectors, $\mathbf{Q}_{1}=$ $(0.25,0.25,3.5)$ and $\mathbf{Q}_{2}=(0.75,0.75,0)$. Neutrons scatter from spin fluctuation components perpendicular to $\mathbf{Q}$, so at $\mathbf{Q}_{1}$ the signal is mainly from in-plane fluctuations, and at $\mathbf{Q}_{2}$ the signal comes equally from in-plane and $c$-axis components. For each $\mathbf{Q}$, we measured the SF intensity with $\mathbf{P} \| \mathbf{Q}$ and with $\mathbf{P} \perp \mathbf{Q}$ in two orthogonal directions. Because SF scattering arises from spin fluctuations perpendicular to $\mathbf{P}$, these measurements allow us to determine the spin fluctuation anisotropy [10]. From the $\mathbf{Q}_{1}$ data, we found that the spin fluctuations are isotropic within the $\mathrm{NiO}_{2}$ layers to within the experimental error of $20 \%$. The $\mathbf{Q}_{2}$ measurements revealed that the $c$-axis component of the magnetic response is a factor $2.3 \pm 0.4$ larger than the in-plane response. In other words, the fluctuating spin components that give rise to the diffuse scattering are anisotropic, with a strong tendency to 
fluctuate towards the crystal $c$ axis. This conclusion is consistent with the observation of a decrease in scattering intensity in the out-of-plane direction mentioned earlier.

What can we infer from the results described thus far for energies of 2-2.5 meV? The existence of ridges of inelastic magnetic scattering running along the diagonals of the $(h, k)$ reciprocal lattice plane implies the existence of dynamic spin fluctuations with a short correlation length along the ridge direction and a relatively long correlation length perpendicular to the ridge. The absence of any out-of-plane modulation in the scattering means the correlations along the $c$ axis are extremely weak. Hence, the spin fluctuations are quasi-1D, with the strongest correlations parallel to the diagonals of the square lattice, i.e., parallel to the stripe directions.

To understand the local pattern of spins responsible for the diffuse scattering, we consider an array of $1 \mathrm{D} \mathrm{AF}$ chains running parallel to the direction $\mathbf{d}=[1,1,0]$ with spins attached to the vertices of the square latticeFig. 1(c). In the absence of interchain correlations, most of the spectral weight is concentrated near the lower bound of the spin excitation spectrum, which in the case of a spin- $\frac{1}{2}$ Heisenberg chain is given by [11]

$$
E(\mathbf{Q})=\pi J|\sin (2 \pi \mathbf{Q} \cdot \mathbf{d})|,
$$

where $J$ is the exchange energy per spin. At vanishingly small energies, the scattering cross section is largest when $\mathbf{Q} \cdot \mathbf{d}$ is half an odd integer, and zero when $\mathbf{Q} \cdot \mathbf{d}$ is an integer. Therefore, we expect the low-energy $(E \ll 4 J S)$ scattering to be strongly peaked along the broken lines shown in Fig. 1(b), which intersect the scan direction marked $\mathrm{A}$ at $\mathbf{Q}=\left(\frac{1}{4}, \frac{1}{4}, 0\right),\left(\frac{3}{4}, \frac{3}{4}, 0\right)$, etc. Similarly, the low-energy scattering from spin chains oriented along the other diagonal $\mathbf{d}^{\prime}=[1,-1,0]$ would lie along the solid diagonal lines in Fig. 1(b).

It can be seen that the distribution of diffuse scattering we have observed is consistent with this model. The main discrepancy is a slight meandering of the diffuse scattering shown in Fig. 1(a). For example, the peak position in the $2 \mathrm{meV}$ scan shown in Fig. 2(a) is not quite at $\zeta=\frac{1}{4}$. Looking in detail, we find that the diffuse scattering closely follows the stripe superlattice zone boundary [7], which suggests the spin chains are weakly coupled and have the same periodicity as the stripes.

We now discuss measurements showing how the diffuse scattering varies with energy and temperature. In Fig. 3(a), we plot some of the raw data from a series of wave vector scans along line $\mathrm{B}$, each scan having a fixed energy. The scan coordinate $\zeta$ corresponds to $\left(\mathbf{Q} \cdot \mathbf{d}^{\prime}\right) / 2$ for a spin chain parallel to $\mathbf{d}^{\prime}$. With increasing energies above $\sim 4 \mathrm{meV}$, one can clearly observe two peaks separating from the $1 \mathrm{DAF}$ wave vector $\zeta=\frac{1}{4}$. In Fig. 3(b), we have plotted the dispersion of these two peaks. The peak centers were obtained by fitting each of the scans in Fig. 3(a) with two Gaussians on a linear background.

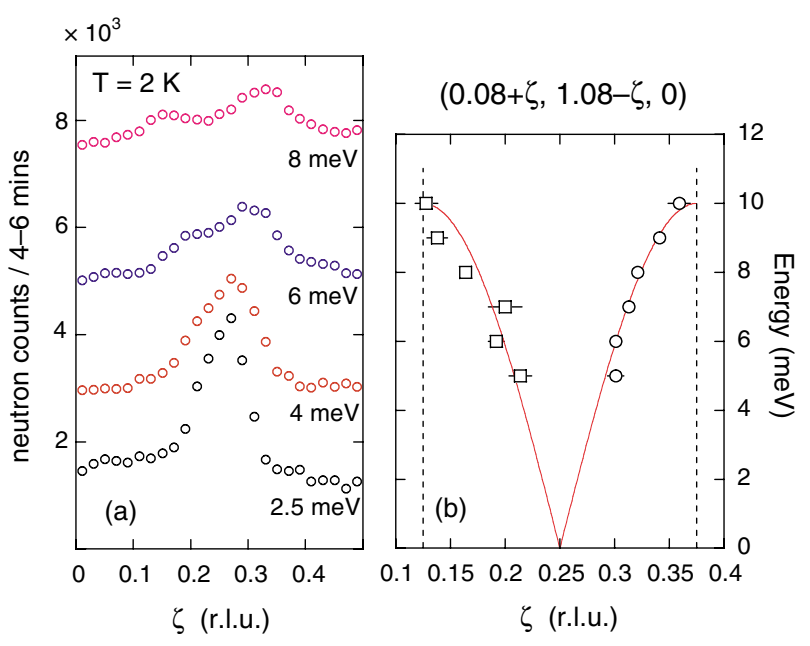

FIG. 3 (color online). (a) Constant-energy scans along the line marked B in Fig. 1(b). Successive scans have been displaced vertically by 2000 counts for clarity. (b) Dispersion of the peak positions from a series of constant-energy scans including those shown in (a). The line is the lower bound to the excitation spectrum of a spin- $\frac{1}{2}$ Heisenberg AF chain calculated from Eq. (1) with $J=3.2 \mathrm{meV}$. The vertical dotted lines indicate one period of the lower bound curve.

The result is consistent with a dispersion described by Eq. (1) with a maximum energy of $10 \mathrm{meV}$.

Figure 4 displays the temperature dependence of diffuse scattering at an energy of $2 \mathrm{meV}$, measured once again in a scan along line B in Fig. 1(b). With increasing temperature, the intensity of the peak decreases and the width increases. These data show that the spin correlations become weaker with temperature, and are almost wiped out above $100 \mathrm{~K}$.

The measurements reported here show that the new spin fluctuations we have found behave very differently from the low-energy spin excitations of the host $\mathrm{Ni}^{2+}$ spins studied previously [7]. The latter are localized in $\mathbf{Q}$, have in-plane polarization below an anisotropy gap of $\sim 5 \mathrm{meV}$, and remain correlated up to the magnetic ordering temperature of $\sim 200 \mathrm{~K}$ [12]. The diffuse scattering, on the other hand, is spread out in $\mathbf{Q}$, shows predominant out-of-plane anisotropy, and survives only to $\sim 100 \mathrm{~K}$.

These differences imply that the diffuse scattering does not arise from the AF-ordered $\mathrm{Ni}^{2+}$ spins located between the charge stripes, but instead originates from AF correlations in the charge stripes themselves. Our central conclusion, therefore, is that there exist quasi1D AF fluctuations among the stripe electrons. These fluctuations are dynamic on the time scale probed by neutron scattering [13], and from the half-width of the diffuse scattering ridge we estimate the correlation length of the fluctuations along the stripes to be $\sim 15 \AA$. The charges are thought to be localized on $\mathrm{Ni}^{3+}$ ions [14], which means they would carry a spin $S=\frac{1}{2}$ in the strong crystal field limit. Hence, we can estimate the AF 


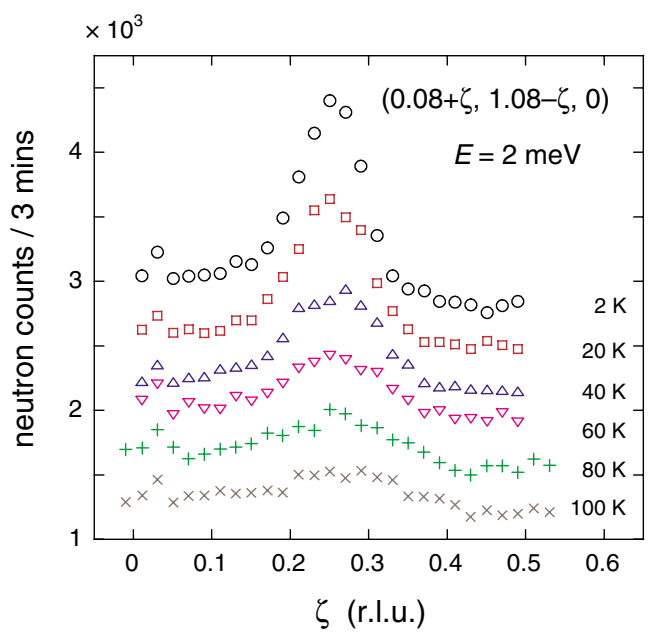

FIG. 4 (color online). Temperature dependence of the diffuse scattering measured in a scan along line B in Fig. 1(b). The energy was fixed at $2 \mathrm{meV}$. Successive scans have been displaced vertically by 400 counts for clarity.

exchange parameter $J$ by fitting Eq. (1) to the data in Fig. 3(b). The result is $J \approx 3.2 \mathrm{meV}$.

Attempts to explore theoretically the properties of stripe phases typically model the stripes as 1D electron liquids coupled via pair hopping or spin exchange to an insulating AF background [3]. These models commonly predict a spin gap in the spectrum of the stripe electrons, allowing various instabilities to occur, including superconductivity, depending on the model parameters. The results described here represent the first measurements of the spin dynamics of stripe electrons, and are important because they provide data with which to constrain the above-mentioned charge-stripe models. Hence, this is a step towards understanding the role of stripes in the problem of cuprate high- $T_{\mathrm{c}}$ superconductivity.

Of course, one should be aware that stripes in the nickelates and cuprates differ somewhat in character. In the nickelates, the stripes are very stable and are aligned along the diagonals of the square lattice, whereas in the superconducting cuprates the stripes are more dynamic and are aligned horizontally and vertically [5]. Also, the 1D band formed by the stripe electrons is half filled in the nickelates (hence, insulating stripes) [15], but is quarter filled in the cuprates (metallic stripes). Nevertheless, the discovery of AF correlations among the stripe electrons in a nickelate (in contrast to theoretical predictions of ferromagnetic stripes [15]) raises general questions about the mechanism of intrastripe spin exchange and about the coupling between the stripe electrons and the surrounding AF region. A grasp of these phenomena in a simple reference material such as $\mathrm{La}_{5 / 3} \mathrm{Sr}_{1 / 3} \mathrm{NiO}_{4}$ could contribute towards a better understanding of the stripe phase in other systems. Looking ahead, our results suggest it would be well worthwhile to conduct a search for spin correlations among the stripe electrons in a stripeordered cuprate.
We thank L.-P. Regnault for help with the experiments on IN22. This work was supported by the Engineering \& Physical Sciences Research Council of Great Britain. This work was performed in part at the Swiss Spallation Neutron Source SINQ, Paul Scherrer Institute (PSI), Villigen, Switzerland.

*Electronic address: a.boothroyd1@physics.ox.ac.uk URL: http://xray.physics.ox.ac.uk/Boothroyd

[1] C. H. Chen, S.-W. Cheong, and A. S. Cooper, Phys. Rev. Lett. 71, 2461 (1993); J. M. Tranquada et al., Phys. Rev. Lett. 73, 1003 (1994); S. Mori, C. H. Chen, and S.-W. Cheong, Nature (London) 392, 473 (1998).

[2] J. Zaanen and O. Gunnarsson, Phys. Rev. B 40, 7391 (1989); K. Machida, Physica (Amsterdam) 158C, 192 (1989); V. J. Emery and S. A. Kivelson, Physica (Amsterdam) 209C, 597 (1993); D. Poilblanc and T. M. Rice, Phys. Rev. B 39, 9749 (1989); H. L. Schultz, J. Phys. (Paris) 50, 2833 (1989).

[3] V. J. Emery, S. A. Kivelson, and O. Zachar, Phys. Rev. B 56, 6120 (1997); Yu. A. Krotov, D.-H. Lee, and AV. Balatsky, Phys. Rev. B 56, 8367 (1997); H. Johannesson and G. I. Japaridze, cond-mat/0301585 [Phys. Rev. B (to be published)].

[4] T. R. Thurston et al., Phys. Rev. B 40, 4585 (1989); S.-W. Cheong et al., Phys. Rev. Lett. 67, 1791 (1991); P. Dai, H. A. Mook, and F. Dŏgan, Phys. Rev. Lett. 80, 1738 (1998); H. A. Mook et al., Nature (London) 404, 729 (2000).

[5] J. M. Tranquada et al., Nature (London) 375, 561 (1995).

[6] S. M. Hayden et al., Phys. Rev. Lett. 68, 1061 (1992); J. M. Tranquada, P. Wochner, and D. J. Buttrey, Phys. Rev. Lett. 79, 2133 (1997); K. Nakajima and Y. Endoh, J. Phys. Soc. Jpn. 67, 1552 (1998); S.-H. Lee et al., Phys. Rev. Lett. 88, 126401 (2002); P. Bourges et al., Phys. Rev. Lett. 90, 147202 (2003).

[7] A. T. Boothroyd et al., Phys. Rev. B 67, 100407(R) (2003).

[8] D. Prabhakaran, P. Isla, and A. T. Boothroyd, J. Cryst. Growth 237, 815 (2002).

[9] S.-W. Cheong et al., Phys. Rev. B 49, 7088 (1994); H. Yoshizawa et al., Phys. Rev. B 61, R854 (2000); C.-H. Du et al., Phys. Rev. Lett. 84, 3911 (2000); S.-H. Lee et al., Phys. Rev. B 63, 060405 (2001).

[10] R. M. Moon, T. Riste, and W. C. Koehler, Phys. Rev. 181, 920 (1969).

[11] J. des Cloizeaux and J. J. Pearson, Phys. Rev. 128, 2131 (1962).

[12] S.-H. Lee and S.-W. Cheong, Phys. Rev. Lett. 79, 2514 (1997).

[13] Preliminary high-resolution measurements show a reduction in diffuse scattering intensity below $\sim 1 \mathrm{meV}$, indicative either of a spin gap of $\sim 0.5 \mathrm{meV}$ or a quasielastic response with a width $\Gamma \sim 1 \mathrm{meV}$.

[14] J. Li et al., Phys. Rev. B 67, 012404 (2003).

[15] J. Zaanen and P. B. Littlewood, Phys. Rev. B 50, 7222 (1994). 